\section{AB0560 DESCRIPTIVE ANALYSIS AND STUDY OF CORRELATIONS IN A COHORT OF PATIENTS WITH INFLAMMATORY MYOPATHIES}

I. Carrión Barberà ${ }^{1}$, A. Pros Simón ${ }^{1}$, T. C. Salman Monte ${ }^{1}$, M. Ciria ${ }^{1}$, F. VílchezOya ${ }^{1}$, S. Labrada ${ }^{1}$, T. Meraz ${ }^{1}$, J. Monfort ${ }^{1} .{ }^{1}$ Hospital del Mar, Barcelona, Spain

Background: Inflammatory myopathies (IM) are a group of rare diseases that involve muscle inflammation. Several types are defined with a wide range of different manifestations and prognosis.

Objectives: Describe the characteristics of the cohort of patients with IM in a tertiary hospital, in order to identify their demographic and clinical characteristics and try to find a correlation between them.

Methods: Retrospective observational study of patients with IM: dermatomyositis (DM), polymyositis, antisynthetase syndrome (ASS), necrotizing autoimmune myopathy and overlap syndrome (OS). Clinical, biological, neurophysio and histopathological data were collected. Statistical analysis was performed using SPSS 23 IBM®.

Results: 28 patients were included with a follow-up of $10.9 \pm 9.8$ years $(y)$. According to the 2017 EULAR / ACR criteria for IM, $89.2 \%$ were classified as definitive $\mathrm{MI}$, with an average score of $12.1 \pm 3.2$. Age at diagnosis $47.3 \pm 17.7 \mathrm{y}$; ratio $1.3 ; 78.6 \%$ Caucasian, $10.7 \%$ Asian and $10.7 \%$ Latino. $39.3 \%$ had DM, $3.6 \%$ hypomyopathic and 3.6\% amyopathic DM, $28.6 \%$ OS and $17.9 \%$ ASS. Lung involvement was the most prevalent extramuscular manifestation (67.9\%). Systemic sclerosis was the most frequent overlapping autoimmune disease (AD) $(21.4 \%)$ and 2 patients $(7.1 \%)$ overlapped more than 1 AD. In Table 1 are detailed the clinical characteristics of the patients, and in Figures 1 and 2, the autoantibody (aa) profile and treatments used. The incidence of neoplasm was $10.7 \%$ $10.3 \pm 9.6 y$ after the diagnosis of myopathy ( 3 breast neoplasms, 1 colon and 1 cutaneous lymphoma), and of them, $66.7 \%$ had two synchronous neoplasms. No neoplasms were observed in the 2 anti-TIF1- $\gamma+$ patients. The subtype of IM in these patients was $1 \mathrm{OS}$ anti-RNP+, $1 \mathrm{DM}$ anti-PL-12+ and 1 ASS anti-OJ+. $17.9 \%$ smoked and $21.4 \%$ had taken statins at some point, without it being related to the start of the myopathic clinic. A capillaroscopy was performed in $67.9 \%$, being pathological in $63.1 \%$

The positivity of anti-RNP $(p=0.01)$ and steroid bolus $(p=0.039)$ were correlated with a more severe disease, defined as a summation index composed of a series of manifestations (pulmonary hypertension $(\mathrm{PH})$, ischemic heart disease, venous/arterial thrombosis, myo/pericarditis, interstitial lung

Table 1. Clinical characteristics.

\begin{tabular}{lcc}
\hline Clinical manifestations & Frequency \% \\
\hline Presentation & Acute & 40.7 \\
& Subacute & 22.2 \\
Muscular Weakness & Insidious & 37 \\
Muscle Enzymes Elevation & & 82.1 \\
Muscle Pain & & 85.7 \\
Joint Manifestations (Mf) & & 67.9 \\
Systemic Mf & & 67.9 \\
Digestive Mf & & 67.9 \\
Raynaud's Syndrome (RS) & & 46.4 \\
Sclerodactyly & & 53.6 \\
Digital ulcers (DU) & & 32 \\
Calcinosis & & 25 \\
ILD & & 10.7 \\
& & 67.9 \\
& Nonspecific Interstitial Pneumonia & 63.2 \\
& Usual Interstitial Pneumonia & 15.8 \\
& Organizing Pneumonia & 10.5 \\
PH & Lymphocytic Interstitial Pneumonia & 5.3 \\
Serious Infections & Undefined Pattern & 5.3 \\
& & 10.7 \\
& & 17.9 \\
\hline
\end{tabular}

Table 2. Correlations between clinical manifestations and aa.

\begin{tabular}{lcc}
\hline Manifestations & Autoantibodies & p value \\
\hline DU & Anti-MDA5 & 0.005 \\
Sclerodactyly & Anti-RNP & 0.011 \\
Pericarditis & Anti-RNP & 0.000 \\
Myocarditis & Anti-RNP & 0.005 \\
Diabetes & Anti-RNP & 0.027 \\
RS & Anti-PM/Scl & 0.033 \\
Calcinosis & Anti-PM/Scl & 0.027 \\
Flexion Contractures & Anti-PM/Scl & 0.027 \\
Arrhythmias & Anti-PL-12 & 0.001 \\
\hline
\end{tabular}

disease (ILD), severe infections, neoplasms or hospitalizations). Other statistically significant correlations between aa and clinical manifestations are detailed in Table 3, among which the anti-RNP+ with myopericarditis stands out. No correlation was found between the findings on capillaroscopy and the type of IM.

Conclusion: The most frequent subtype of IM was DM. $10.3 \%$ of the patients presented a neoplasm, all with different subtypes of myopathy and aa. The presence of anti-RNP+ correlated with greater severity of the disease and myopericarditis. Likewise, significant differences were found between the subtypes of aa and certain clinical manifestations. There is no correlation between findings on capillaroscopy and the type of IM.

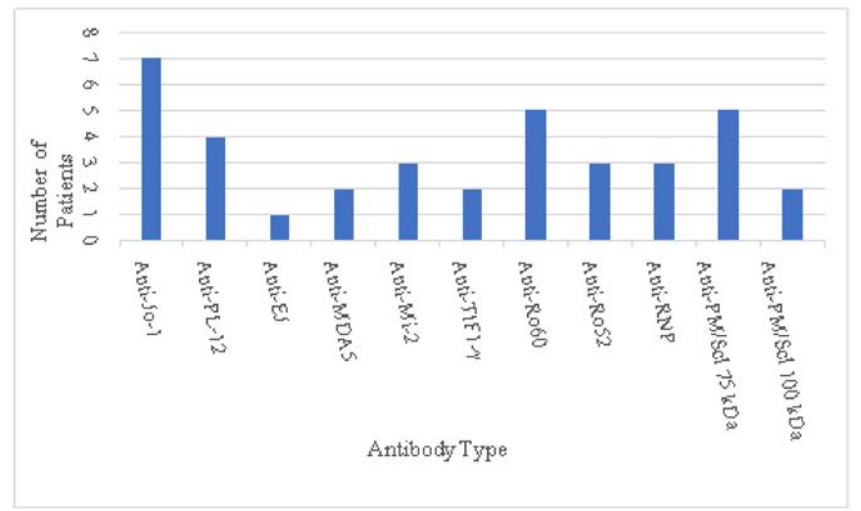

Figure 1. Aa.

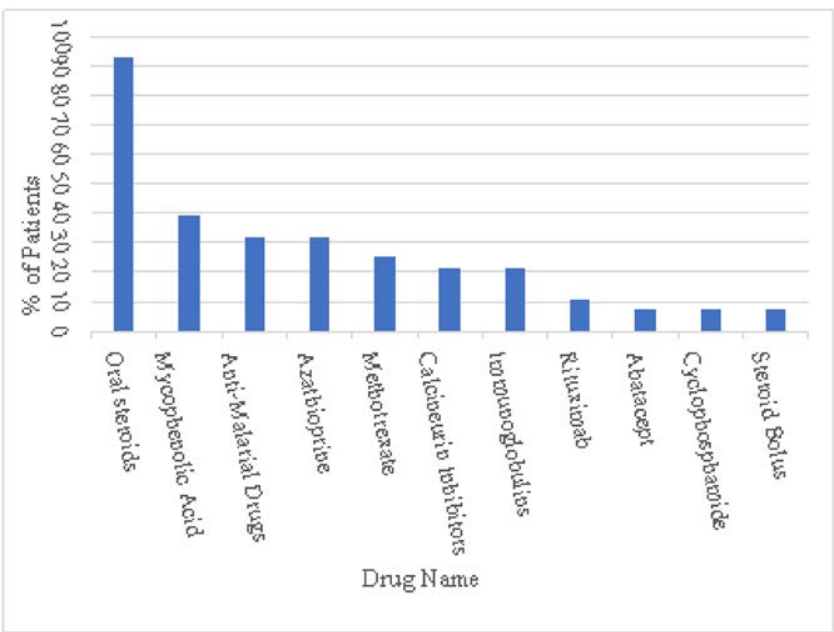

Figure 2. Treatment.

Disclosure of Interests: Irene Carrión Barberà Grant/research support from: I received a grant from the Spanish Rheumatology Foundation (FER) and laboratories KERN PHARMA for a brief stay abroad., Ana Pros Simón: None declared, Tarek Carlos Salman Monte: None declared, Manel Ciria: None declared, Francisco Vílchez-Oya: None declared, Selene Labrada: None declared, Toni Meraz: None declared, Jordi Monfort: None declared DOI: 10.1136/annrheumdis-2020-eular.2899

\section{$\mathrm{AB} 0561$ \\ AUTOMATIC QUANTIFICATION OF INTERSTITIAL LUNG DISEASE FROM CHEST COMPUTED TOMOGRAPHY IN SYSTEMIC SCLERODERMA}

A. Carvalho ${ }^{1}$, A. Guimarães ${ }^{2}$, F. R. Sztajnbok ${ }^{3}$, R. Rodrigues ${ }^{4}$, B. Silva ${ }^{5}$, A. Lopes ${ }^{5}$, I. Almeida ${ }^{6}$, M. França ${ }^{7} .^{1}$ Medical School, Hosp. de Santo António (HSA), Centro Hospitalar do Porto (CHP), Instituto de Ciências Biomédicas Abel Salazar (ICBAS), Univ. do Porto, Department of Radiology, Porto, Portugal; ${ }^{2}$ Laboratory of Pulmonary Engineering, Biomedical Engineering Program, Alberto Luiz Coimbra Institute of Post-Graduation and Research in Engineering, Universidade Federal do Rio de Janeiro, Rio de Janeiro, Brazil; ${ }^{3}$ Department of Reumathology, State University of Rio de Janeiro, Rio de Janeiro, Brazil; ${ }^{4}$ Department of Radiology, Universidade Federal do Rio de Janeiro, Rio de Janeiro, Brazil; ${ }^{5}$ Postgraduate Program in Medical Sciences, School of Medical 